\title{
Optimizing Road Milling and Resurfacing Actions
}

\author{
Mariem Zouch, Thomas Yeung and Bruno Castanier \\ Department of Automatic control \& Industrial Engineering \\ Ecole des Mines de Nantes / IRCCyN , F-44307 NANTES Cedex, France
}

\begin{abstract}
A condition-based maintenance optimization approach is developed for the road-cracking problem in order to derive optimal maintenance policies that minimize a total discounted maintenance cost. The approach is based on a Markov decision process that takes into account multiple actions with varying effects on future road performance. Maintaining the road consists of adding a new asphalt layer; however, as resurfacing actions are constrained by a maximum total road thickness, the maintenance decision is not only how thick a layer to apply, but also how much old road to remove. Each combination of these actions leads to different maintenance costs and different future degradation behaviours. The road state is modelled by a dependent bivariate deterioration variable (the longitudinal cracking percentage and the deterioration growth rate), for taking these different changes in the cracking patterns into account. Moreover, the sensitivity to cracking for existing roads can be reduced with the addition of new layers, and thus actions that can lead to states better than good-as-new have to be considered. A numerical analysis is provided to illustrate the benefits of the introduction of the deterioration speed in the decision framework, as well as the belief that initially building a road to its maximum thickness is not optimal. The trade-offs in the design decisions and the exploitation/maintenance costs are also explored.
\end{abstract}

Keywords: road cracking, stochastic deterioration, maintenance optimization, Markov decision processes 


\section{Notations}

$\begin{array}{ll}c_{0} & \text { inspection cost } \\ c_{1} & \text { fixed costs of milling } \\ c_{2} & \text { fixed costs of resurfacing } \\ c_{a}(\xi) & \text { variable cost of adding thickness } \xi \\ & \\ c_{r}(\zeta) & \text { variable cost of removing thickness } \zeta \\ & \\ c_{q}(\cdot) & \text { quality cost } \\ f(\cdot) & \text { density function of } \theta \text { in both phases } \\ g(\cdot) & \text { density function of } \rho \text { in both phases } \\ h(\cdot) & \text { joint }(\rho, \theta) \text { density function in both } \\ & \text { phases } \\ i_{\theta} & \text { discrete value of } \theta \\ i_{\rho} & \text { discrete value of } \rho \\ N_{\theta} & \text { number of possible values of } \theta \\ N_{\rho} & \text { number of possible values of } \rho \\ S & \text { set of states } \\ s & \text { state of the section } \\ V & \text { Total expected discounted cost }\end{array}$

$\alpha(\cdot) \quad$ cumulative hazard function of the Poisson process

$\ell \quad$ section thickness

$\ell_{\max }$ maximum section thickness

$\gamma_{1}(\cdot)$ linear-form shape function of the state-dependent gamma process gaussian-form shape function of the state-dependent gamma process length of discrete intervals of $\theta$ length of discrete intervals of $\rho$ milling thickness deterioration growth rate

$\theta_{\max }$ maximum growth rate

$\lambda$ discount factor

$\xi_{m} \quad$ last added thickness

$\pi \quad$ maintenance policy

$\rho \quad$ longitudinal cracking percentage

$\tau \quad$ decision epoch length

$\xi \quad$ resurfacing thickness

$\phi(\cdot)$ maintenance effect function on the deterioration growth rate

\section{Introduction}

During recent decades, transportation administrations of several countries have made continuing efforts to develop and implement efficient pavement management systems (PMS) in order to define the best maintenance policies that ensure acceptable use and safety conditions of the road in the most cost-effective way possible. A survey of existing PMSs is presented in reference [2]. According to reference [9], a PMS consists of four main components: the inventory, the pavement condition evaluation, the performance prediction models, and the planning method. Definition of the deterioration model is one of the most important components, as it determines the quality and the efficiency of the optimal policy.

From a survey of maintenance optimization approaches [1, 12], deterioration models can be classified into three classes as a function of the consistency of information and knowledge on the deteriorating process. The first class consists of models based on a resistance-load relationship, such as the Paris-Ergodan equation [6]. They have proved their efficiency for reliability purposes, but because of their numerical complexity they become intractable in more maintenance decision frameworks. The second class consists of models based on Markov processes [3, 4], which are widely used in existing PMSs. These models require the determination of a transition probability matrix, usually obtained by statistical data analysis techniques [2] when large amounts of deterioration data are available, or by expert judgements when only a few data are available. The third class contains models based on Lévy processes, such as 
the Brownian motion and gamma processes that are widely used for cumulative deterioration modelling [11]. Zouch et al. [13] proposed a deterioration model that is a combination of the two last model classes.

Moreover, existing cracking models are based on observable deterioration indexes that only model the observable deterioration through quality indexes such as the surface distress index, the distress manifestation index, or the pavement condition index [2], and do not take into account the underlying deterioration processes. In fact, most of the existing conditionbased approaches utilize a restrictive definition of the system state classically defined by an observable and measurable metric such as the size of the road cracks. This can be restrictive, especially when modelling the effects of imperfect maintenance. Different imperfect actions can have the same effects on the observable deterioration, i.e. concealing the observable damage, but different effects on the level of both the underlying and future deterioration.

Few maintenance planning methods, as in references [8] and [10] consider imperfect maintenance actions in addition to minimal and perfect repairs. In these approaches, the effects of imperfect maintenance consist of a partial reduction in the deterioration level, but they do not allow for the change of the system deterioration law, as in reference [13]. In the latter paper, Zouch et al. present a condition-based maintenance optimization approach for road maintenance that takes into account multiple imperfect actions with different effects on the immediate state of the road, as well as its future deterioration law. This is made possible by considering a second deterioration parameter that models the underlying deterioration process in addition to the observable parameter. The two parameters are then used to define a "state-dependent" deterioration model, where the deterioration law in each decision period depends on the deterioration level just before maintenance and the performed action.

To derive the optimal maintenance policy that minimizes the discounted total cost over the infinite horizon, a classical dynamic programming formulated as a Markov decision process (MDP) [7] is solved. The state-dependent deterioration model is incorporated into the MDP framework to derive action-dependent transition matrices.

In reference [9], maintenance decisions relate to the road thickness to renew, i.e. remove and add. The total road thickness is therefore kept fixed. The objective of this paper is to extend the model presented in reference [13] to take into account a constraint of a maximum road thickness, in order to make the model more realistic and more applicable. The maximum thickness constraint renders the decision more complex, as it consists in determining not only the resurfacing layer thickness to add, but also the thickness to remove prior to resurfacing. A special feature of this model is that consecutive changes in road thickness and composition, i.e. in new and old layers, may result in a better-than-new road performance. The possibility to restore a system to a state better than as-good-as-new (AGAN) is a new aspect in the maintenance literature. Our model accounts for the reality that two roads of the same thickness are not equal, and that in general the road with more layers will be superior.

The remainder of the paper is organized as follows. Section 2 briefly presents the statedependent deterioration model for the road longitudinal cracking process. The optimization problem is formulated as a Markov decision process in Section 3. Finally, Section 4 is dedicated to the solution procedure and sensitivity analysis from numerical examples. 


\section{The road cracking process}

Road deterioration is essentially due to harsh environmental conditions and traffic loads. One of the most important deterioration modes is longitudinal cracking, as it represents the structural health of the road. Meyers et al. [5] reported that over 90 percent of road sections in Florida that are in need of repair have such cracks. The longitudinal cracking process operates as follows. A repetitive tensile stress is generated at the bottom of the asphalt layer, and leads the road's tensile strength to deteriorate over time. When the stress applied by the traffic load exceeds the tensile strength of the road, micro-cracks appear at weak spots in the base of the road. These micro-cracks then propagate through the inferior layers of the road until they reach the road surface to give way to surface cracks that continue to propagate. The road-cracking process is therefore a two-phase cumulative process. The first phase is the initiation phase, during which no deterioration can be observed on the surface, but the road cannot be considered to be in perfect condition. The second phase is the propagation phase, which begins with the arrival of the first observable crack.

The current metric used in France to measure the cracking level of a road section is the longitudinal cracking percentage (LCP), represented in Figure 1. Note that the LCP met-

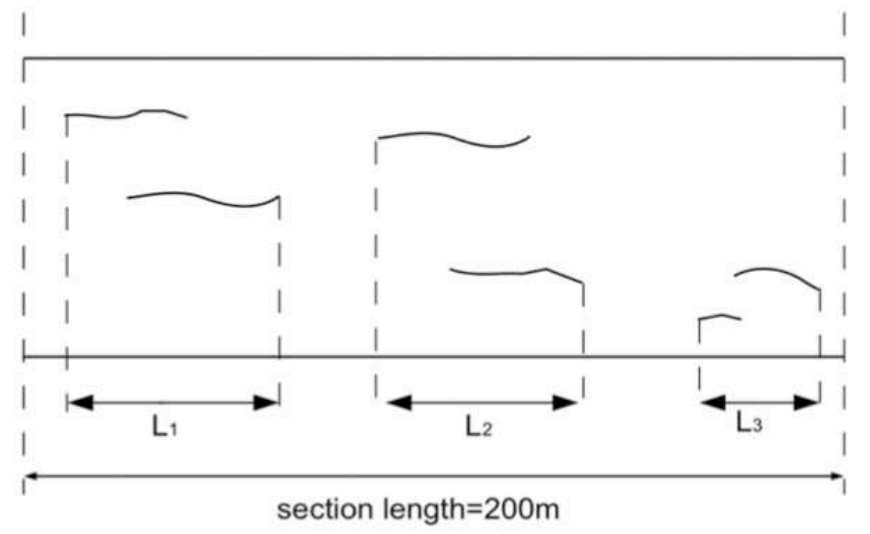

Figure 1: Longitudinal cracking percentage metric for $200 \mathrm{~m}$ road section $\mathrm{LCP}=\frac{L_{1}+L_{2}+L_{3}}{200}$

ric represents only the total longitudinal cracking, and does not take into account the number of cracks or overlapping cracks. Moreover, it is not suitable for the initiation phasewhere no cracks are observable. In reference [13], Zouch et al. have highlighted the benefits of introducing a new decision parameter, the deterioration growth rate (DGR), which models the underlying cracking process. More specifically, the DGR models a cracking potential in the first phase when the road is crack-free, and an instantaneous speed of deterioration in the second phase.

In this paper, the two-phase deterioration model developed in reference [13] is used. More specifically, the LCP and DGR are modelled as stochastic processes, denoted by $\left\{\rho_{t}\right\}_{t \geq 0}$ and $\left\{\theta_{t}\right\}_{t \geq 0}$, respectively. In the first phase, the cracking potential $\mathrm{u}$ and the first observable crack arrival are considered and modelled as a gamma process and a $\theta$-dependent Poisson process, respectively. In the second phase, the DGR $\theta$ and the LCP $\rho$ are modelled using a bilateral 
gamma (BG) process and a state-dependent gamma (SDG) process, respectively. The proposed gamma process in reference [13] is called state-dependent, since its shape function in each decision epoch depends on the level of deterioration at its start, given by $(\rho, \theta)$ and on the last performed action. For more details of the deterioration model and the SDG process, the reader should refer to reference [13].

\section{Model Formulation}

We consider a road section, characterized by an initial thickness $\ell$, that is continuously and stochastically deteriorating under the two-phase deterioration process [13] presented in the previous section. Periodic inspections are performed at the beginning of the decision epoch of length $\tau$ to yield perfect observations of the LCP $\rho$. Since $\theta$ cannot be measured, the problem is partially observed. However, the DGR $\theta$ can be approximated in the propagation phase using successive observations of $\rho$, and estimated in the initiation phase [13]. The problem can therefore be solved as a fully observable problem.

Based on the information yielded by inspection, the decision-maker should decide whether to do nothing $(\mathrm{DN})$ and let the system deteriorate until the next decision epoch, or to maintain the section $(\mathrm{MX})$. As the road thickness is constrained by a maximum thickness $l_{\max }$, the maintenance consists of milling a thickness $\zeta$ before adding a new resurfacing layer of thickness $\xi$. Resurfacing the road section with a new layer conceals the longitudinal cracks, so that $\rho$ is reset to zero, but also changes the thickness and the composition of the section, which makes it more rigid and reduces its DGR $\theta$.

A maintenance decision $(\xi, \zeta)$ incurs an action cost $c_{1}+c_{2}+c_{r}(\zeta)+c_{a}(\xi)$, where $c_{1}$ and $c_{2}$ are the fixed costs of removing and adding, respectively, whereas $c_{r}(\zeta)$ and $c_{a}(\xi)$ are the variable costs of removing a thickness $\zeta$ and adding a thickness $\xi$.

Moreover, a quality cost $c_{q}(\cdot)$ is incurred for beginning a decision epoch in a given state $(\rho, \theta, \ell)$. This quality cost represents a penalty or a risk function for leaving the road in a deteriorated state for the current decision epoch.

Both decisions and maintenance actions are performed instantaneously at the beginning of each decision epoch, as decision and maintenance times are assumed to be negligible compared with the decision epoch length. The state of the road section is defined by $\left(\rho, \theta, \ell, \xi_{m}\right)$, where $\rho$ and $\theta$ are the current LCP and DGR yielded by inspection, respectively; $\ell$ is the current thickness of the road section; and $\xi_{m}$ is the last thickness added to the road section. Considering the last-performed maintenance action as a state parameter allows the decisionmaker to know the current composition of the road, i.e. the old and new layer thicknesses. Therefore the state of the section is Markovian.

Let $S=[0,1] \times\left[\theta_{0}, \theta_{\max }\right] \times\left[0, \ell_{\max }\right] \times\left[0, \ell_{\max }\right]$ denote the state space. A DN action is assumed to have no impact onto the state of a road section. If a maintenance action $(\xi>0, \zeta>0)$ is selected, the state is transformed into $(0, \phi(s, \xi, \zeta), \ell+\xi-\zeta)$, where $\phi(s, \xi, \zeta)$ is the function that models the deterministic effect of the maintenance action on the DGR. Note that as maintenance actions may change the road section thickness and composition, it is possible to obtain a road that has better performance than the initial one. Thus it is possible to obtain a state better-than-new. 
The objective is to derive an optimal maintenance policy $\mathrm{p}$ that minimizes the total discounted cost-to-go over the infinite horizon, denoted by $V$. Before presenting the MDP formulation, let $g\left(x ; \rho, \theta, \ell, \xi_{m}\right)$ and $f\left(y ; \rho, x, \theta, \ell, \xi_{m}\right)$ denote the density distributions of the LCP and DGR processes in the two phases, respectively, given the current levels of $\rho$ and $\theta$ as well as the current section thickness $\ell$ and the last performed action type given by $\xi_{m}$.

More specifically, $g$ represents the density of the Poisson process in the first phase and the density of the SDG process in the second phase, whereas $f$ represents the density of the BG process in both phases. The joint density of the two deterioration parameters in both phases, denoted $h\left(x, y ; \rho, \theta, \ell, \xi_{m}\right)$ can be expressed as follows:

$$
\begin{aligned}
h\left(x, y ; \rho, \theta, \ell, \xi_{m}\right)= & g\left(x ; \rho, \theta, \ell, \xi_{m}\right) f\left(y ; \rho, x, \theta, \ell, \xi_{m}\right), \\
& x \in[0,1-\rho], y \in\left[-\theta, \theta_{\max }-\theta\right]
\end{aligned}
$$

Explicit expressions of the density distribution functions $g$ and $f$ are given in reference [4]. Hence the MDP formulation of the maintenance optimization problem can be expressed as follows.

For $s=\left(\rho, \theta, \ell, \xi_{m}\right)$,

$$
V^{*}\left(\rho, \theta, \ell, \xi_{m}\right)=c_{0}+\min \left\{D N\left(\rho, \theta, \ell, \xi_{m}\right), M X\left(\rho, \theta, \ell, \xi_{m}\right)\right\}
$$

where

$$
\begin{array}{r}
D N\left(\rho, \theta, \ell, \xi_{m}\right)=\lambda \int_{0}^{1-\rho} \int_{-\theta}^{\theta_{\max }-\theta} h\left(x, y ; \rho, \theta, \ell, \xi_{m}\right) \\
V\left(\rho+x, \theta+y, \ell, \xi_{m}\right) d y d x
\end{array}
$$

and

$$
M X\left(\rho, \theta, \ell, \xi_{m}\right)=\min _{(\xi, \zeta)}\left\{\begin{array}{c}
c_{1} I_{(\zeta>0)}+c_{2} I_{(\xi>0)}+c_{r}(\zeta)+c_{a}(\xi)+ \\
D N\left(0, \phi\left(\rho, \theta, \ell, \xi_{m}, \xi, \zeta\right), \ell+\xi-\zeta, \xi\right)
\end{array}\right\}
$$

subject to

$$
\ell+\xi-\zeta<\ell_{\max }
$$

where $\lambda \in[0,1]$ is the discount factor.

Note that the last added thickness jm is directly introduced as a parameter of the joint density because of its influence in the cracking process, and thus the associated definition of the road state. Equation (1) states that following the DN action when the current state is $s$ incurs a quality cost plus the expected cost-to-go of the system deterioration from the state $s$ to all possible states $s^{\prime}$. The MX action in equation (2) incurs a maintenance cost, plus the quality cost and the cost-to-go of the the system beginning in the resulted state $\left(0, \phi\left(\rho, \theta, \ell, \xi_{m}\right), \ell+\xi-\zeta, \xi\right)$. The thickness constraint is formulated in (4). 


\section{Solution procedure and numerical examples}

\subsection{Solution procedure}

We solve the MDP problem formulated above using the policy iteration algorithm (PIA) [7]. The following discretization of the decision problem is proposed. Assume that $\rho$ and $\theta$ represent the first values in $N_{\rho}$ and $N_{\theta}$ equal-sized discrete intervals of length $\Delta_{\rho}$ and $\Delta_{\theta}$ on $[0,1]$ and $\left[0, \theta_{\max }\right]$, respectively, such that $N_{\rho}$ and $N_{\theta}$ divide evenly into 100 . As $\rho=0$ is a key state, we consider it as a single state. Let $N$ be the cardinality of the state space $S$, and $p_{s s^{\prime}}^{m}$ denote the transition probability from state $s$ to state $s^{\prime}$ when the last performed maintenance type is $m$.

$$
\begin{aligned}
p_{s s^{\prime}}^{m} & =\operatorname{Pr}\left\{s^{\prime} \mid s\right\} \\
& =\operatorname{Pr}\left\{\rho^{\prime} \in\left[i_{\rho^{\prime}}, i_{\rho^{\prime}}+\Delta_{\rho}\right], \theta^{\prime} \in\left[i_{\theta^{\prime}}, i_{\theta^{\prime}}+\Delta_{\theta}\right] \mid \rho=i_{\rho}, \theta=i_{\theta}, \ell, \xi_{m}\right\} \\
& =\int_{i_{\rho^{\prime}}-i_{\rho}+\Delta_{\rho}}^{i_{\rho^{\prime}}-i_{\rho}} g\left(x ; i_{\rho}, i_{\theta}, \ell, \xi_{m}\right) \int_{i_{\theta^{\prime}}-i_{\theta}+\Delta_{\theta}}^{i_{\theta^{\prime}}-i_{\theta}} f\left(y ; i_{\rho}, x, \ell, \xi_{m}\right) d y d x
\end{aligned}
$$

The set of possible maintenance actions in order to use the PIA is discretized as follows:

$$
A=\left\{(\xi, \zeta) \mid \xi \in\{5,10,15,20\}, \zeta \in\left[\min \left(0,{ }_{\text {max }}-\ell-\xi\right), \ell\right]\right\}
$$

\subsection{Characterization of the deterioration functions}

We shall focus in this section on the construction of the deterioration function $f$ for the $\rho$ process. The direct derivation of $g$ for the BG process $\left(\theta_{t}\right)$ from the SDG law is proposed in reference [13]. The construction of these laws is expert-based knowledge on the cracking process rather than field data; one of the main motivations is the lack of confidence in the current French database for these longitudinal cracks. Recall that the $\rho$-process is modelled by an SDG process [13], where the deterioration function $f$ on a time interval $t$ is a gamma density function with a shape function, a function of the current state $(\rho, \theta)$, and a given scale parameter $\beta$. Two choices of shape function for $f$ are proposed here.

\subsubsection{The shape function of the SDG process}

For the cracking process $\left(\rho_{t}\right)$, two examples of the SDG process shape function are considered. The first shape function, $\gamma_{1}$, has a linear form of the state parameters, whereas the second function, $\gamma_{2}$, has a form inspired by the well-known Gaussian function:

$$
\begin{gathered}
\gamma_{1}\left(\tau ; \rho, \theta, \ell, \xi_{m}\right)=\left(a_{0}+a_{1} \rho+a_{2} \theta+\frac{a_{3}}{\ell}+\frac{a_{4}}{\xi_{m}}\right) \\
\gamma_{2}\left(\tau ; \rho, \theta, \ell, \xi_{m}\right)=\frac{b_{1}\left(\ell_{\max }-\xi_{m}\right)^{2}}{\ell_{\max }-\ell} \theta^{2} \tau \exp \left\{\frac{(\rho-(1-\theta))^{2}}{b_{2}\left(\ell_{\max }-\xi_{m}\right)}\right\}
\end{gathered}
$$

Note that the two shape functions reflect both time and state effects, as they depend on the decision epoch length $t$ as well as on the state parameters. However, they vary differently as 
the state varies. Figure 2 shows the variations of the expected deterioration in $\rho$ for different levels of $\theta$ given by the Gaussian form shape function. Note that at the beginning of the propagation phase the expected increase in deterioration increases as $\rho$ increases, but with decelerated variation. However, when the DGR level becomes higher, the expected increase in cracking level increases and then decreases quickly, as the DGR is influential. This reflects the fact that, for a single road section, the probability of increasing the cracking level of the section is higher at the beginning of the propagation phase, and decreases as the section becomes more cracked (since overlapping cracks do not account for $\rho$ ). The main objective in considering these two shape functions is to highlight the state-dependent character of the deterioration model, as well as the effect of introducing the DGR as a deterioration parameter.
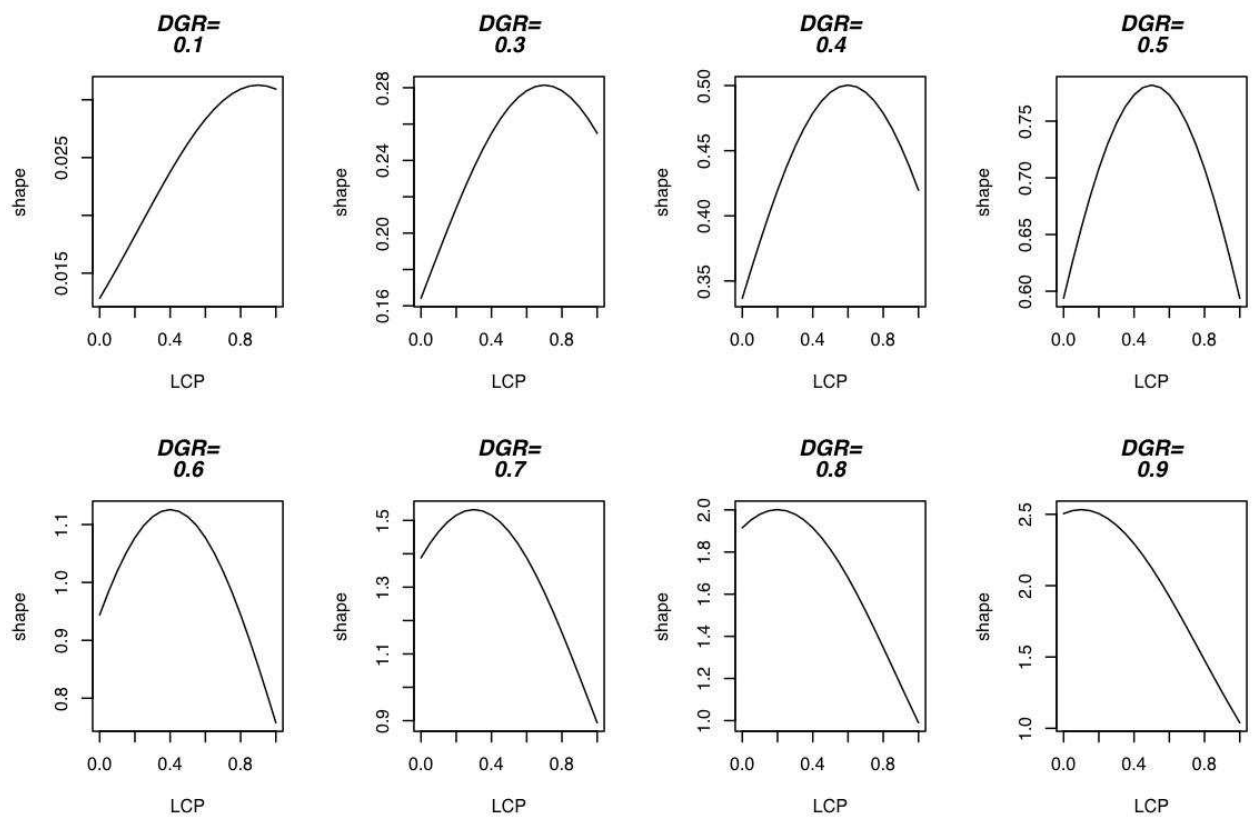

Figure 2: Variations of Gaussian form shape function in $\rho$ for different levels of $\theta$

Figure 3 reflects the fact that the expected crack growth obtained with the Gaussian form shape function increases with the DGR (a), and decreases as the total road thickness and the latest added thickness increase ((b) and (c)).

Figure 4 illustrates the BG process shape functions that represent the expected variation in the DGR $\theta$ as a function of the LCP. Note that the cracking process is accelerated for low LCP levels, i.e. the beginning of the cracking process, and is decelerated when the cracking level is high. This reflects the same behavior shown by the shape function $\gamma_{2}$ of a non-increasing probability of cracking propagation as the section becomes more cracked. 
(a)

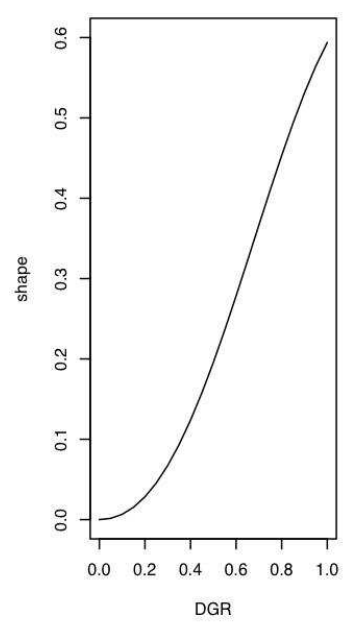

(b)

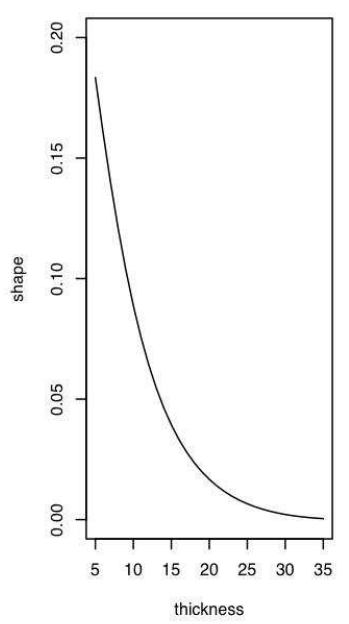

(c)

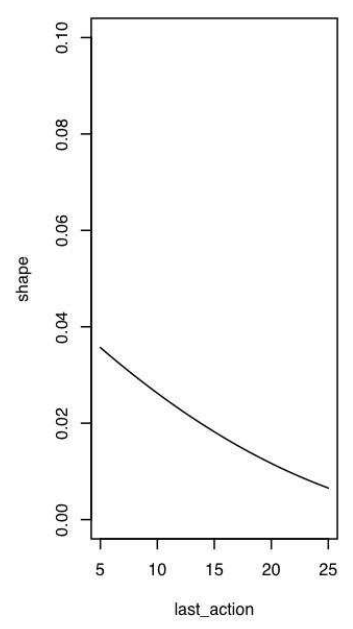

Figure 3: Variations of the Gaussian form shape function in $\theta, \ell$ and $\xi_{m}$
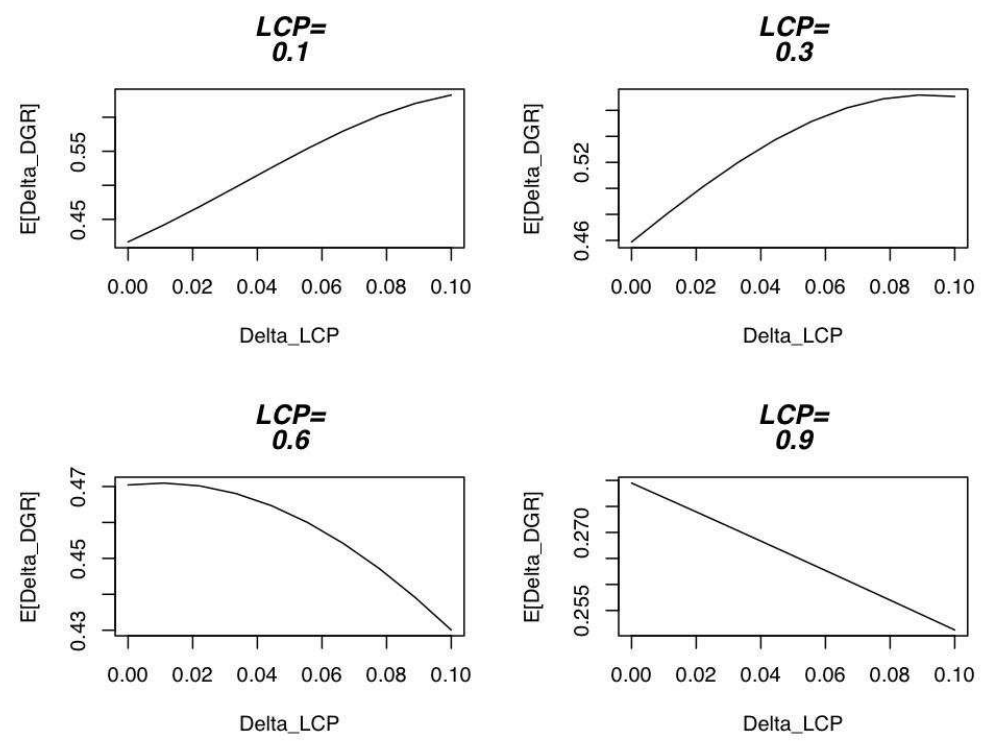

Figure 4: Variations of the DGR in increments of LCP for different levels of $\rho$ 


\subsubsection{Comparison of the linear and Gaussian form shape functions}

The main motivation behind comparing the linear and Gaussian form shape functions is to highlight the state-dependent character of the road-cracking process. In fact, in this model, the deterioration law changes in each decision epoch according to the road state at its beginning and the last performed maintenance action. Both linear and Gaussian form shape functions track this change, since their parameters are state and action dependent. The main difference between the two functions is the fact that, for a given DGR level, the linear shape function has the same variation rate for all LCP levels (the same slope), whereas the Gaussian form function has a variation rate that depends on the cracking level (the slope changes as the LCP changes) (Figure 2).

Moreover, as illustrated in Figure 2, for different values of $\theta$, the linear shape function has a constant variation rate in $\rho$, whereas the Gaussian form function allows for different variation rates in $\rho$ when the DGR level changes. These properties will be illustrated with the numerical analysis to show that the Gaussian form shape function takes future deterioration effects into account better, since it allows changing deterioration speeds for different cracking levels.

\subsubsection{The crack occurrence rate function}

The cumulative hazard function of the Poisson process of the initiation phase is given by the $\theta$-dependent function $\alpha\left(\tau ; \theta, \ell, \xi_{m}\right)$. Note that $\alpha$ is non-decreasing in the cracking potential $\theta$ and non-increasing in the total and new added thicknesses.

$$
\alpha\left(\tau ; \theta, \ell, \xi_{m}\right)=\left(d_{0}+d_{1} \theta+\frac{d_{2}}{\ell}+\frac{d_{3}}{\xi_{m}}\right)
$$

\subsection{Numerical examples}

In the following section, we present some numerical examples, and provide some analysis of the structural properties of the optimal policies.

\subsubsection{Description of the case studies}

We derive optimal policies for two types of section - sections with the current thickness less than the maximum thickness, and sections at maximum thickness - in order to show the effect of the thickness on the road performance as well, as to analyse the trade-off between thicker and newer roads. We consider three sections, which we denote Sec1, Sec2 and Sec3, and which have the following characteristics: $(\ell=10, \xi=10),(\ell=20, \xi=10)$ and $(\ell=30, \xi=5)$, respectively, where each pair denotes the total section thickness $\ell$ and the newer layer thickness $\xi$. 
For $\ell_{\max }=30$, consider the following set of available actions for each section:

$$
\begin{aligned}
& A(\text { Sec } 1)=\left\{(\xi, \zeta)=\left\{\begin{array}{c}
(0,0) ;(10,5) ;(10,10) ;(0,5) ;(0,10) ;(10,15) ; \\
(0,15) ;(10,20) ;(0,20) ;(5,25) ;(10,30)
\end{array}\right\}\right. \\
& A(\text { Sec } 2)=\left\{\begin{array}{c}
(0,0) ;(20,5) ;(10,5) ;(20,10) ;(10,10) ;(0,5) ;(15,15) ; \\
(0,10) ;(20,20) ;(5,15) ;(10,20) ;(15,25) ;(20,30)
\end{array}\right\} \\
& A(\text { Sec } 3)=\left\{\begin{array}{c}
(0,0) ;(25,5) ;(15,5) ;(5,5) ;(30,10) ;(20,10) ;(25,15) ; \\
(10,10) ;(30,20) ;(15,15) ;(20,20) ;(25,25) ;(30,30)
\end{array}\right\}
\end{aligned}
$$

Note that an action can decrease, increase or leave unchanged the total thickness of the road. The efficiency of a maintenance action is defined by a maintenance effect function that reflects the effect of different road compositions (i.e. new and old thick composition) on its performance. An example of a maintenance effect function is given by the following, where $\left(\rho, \theta, \ell, \xi_{m}\right)$ represents the state parameters just before maintenance, and $(\zeta, \xi)$ is the selected maintenance action.

$$
\phi\left(\rho, \theta, \ell, \xi_{m}\right)= \begin{cases}0.85 A \theta & , \xi<\zeta, \xi \geq \xi_{m} \\ 0.75 A \theta & , \xi<\zeta, \xi<\xi_{m} \\ 0.55 A \theta & , \xi=\zeta, \xi \geq \xi_{m} \\ 0.45 A \theta & , \xi=\zeta, \xi<\xi_{m} \\ 0.25 A \theta & , \xi \geq \zeta, \xi \geq \xi_{m} \\ 0.1 A \theta & , \xi \geq \zeta, \xi<\xi_{m}\end{cases}
$$

where $A=\left(\rho+\frac{1}{(\ell+\xi-\zeta)^{2}}+\frac{2}{\xi}\right)$

Note that the maintenance actions in the proposed sets $\mathrm{A}(\operatorname{Sec} 1), \mathrm{A}(\mathrm{Sec} 2)$ and $\mathrm{A}(\mathrm{Sec} 3)$ are ordered in terms of efficiency with respect to this function. We use different quality cost functions that are non-decreasing in deterioration parameters $\rho$ and $\theta$, and non-increasing in the total section thickness and in the last added layer thickness.

\subsection{Numerical analysis}

In the following, we present some optimal policies derived by the PIA for the three considered sections (Sec1, Sec2 and Sec3) using the different input functions presented above.

The deterioration parameters used for the numerical examples are given by $\left(a_{0}, a_{1}, a_{2}, a_{3}, a_{4}\right)=(1,1.5,1.2,1,1), \quad\left(b_{1}, b_{2}\right)=(1.2,2)$

$\left(d_{0}, d_{1}, d_{2}, d_{3}\right)=(1,1.2,1,1)$

The set-up maintenance costs are

$$
c_{1}=900, c_{2}=1700
$$

$c_{r}(\zeta)=c_{u r} \zeta$ with $c_{u r}=90$ the milling cost per thickness unit, $c_{a}(\xi)=c_{u a} \xi$ with $c_{u a}=210$ the resurfacing cost per thickness unit. 
The policies presented in Tables 1-6 state, for each possible road section, the optimal maintenance action to perform. The policies are called Gaussian form and linear form with respect to the shape function used. For example, if the observed section state is $(\rho=0.4, \theta=$ $0.7, \ell=20, \xi_{m}=10$ ), then the action advised by the Gaussian-form policy (Table 1(a)) is resurfacing the road section with a $10 \mathrm{~cm}$ layer without milling, whereas the policy advised by the linear-form policy (Table $1(\mathrm{~b})$ ) is resurfacing the section with a $5 \mathrm{~cm}$ layer without milling.

The discount factor used for the following numerical examples is 0.95 . However, in order to highlight the difference between the two shape functions, some policies with different discount factors are compared. It is found that Gaussian-form policies with a low discount factor (0.3) may converge to linear-form policies obtained with higher discount factors (0.8). This shows that the Gaussian-form shape function takes into account the effects of future deteriorations better than the linear-form function does.

Tables 1-6 present decision matrices that detail the optimal maintenance action for each possible observed state. For example, from Table 1(a), if after inspection Sec2 is found with $\rho=0.3$ and $\theta=0.5$, then the optimal action given by the decision matrix for this state is to resurface the section with a thickness 5 .

The obtained policies present some monotonic properties. More specifically, the efficiency of the recommended action is increasing in the deterioration level and the optimal policy is of the control limit type. For example, in Table 2(a), the DN action is the optimal decision for weak degradation levels (bottom left-hand side of the matrix). When degradation is growing (from the bottom left-hand to the upper right-hand side of the decision matrix), the recommended actions become stronger in terms of costs and efficiency.

The Gaussian-form policies promote predictive policies, whereas the linear form policies do not advise maintenance when the crack level is zero (see the first line of the two matrices in Table 1). This proves that the Gaussian-form shape function takes the effects of the DGR as a deterioration parameter into account better. To show the sensitivity of the optimal policies to the section thickness as well as to its composition, the policies of Tables 1 and 2 are compared, as follows.

1. For thinner sections such as Sec1, note that only actions that increase the section thickness are optimal (Table 2(a)).

2. When the sections are at their maximum thickness, it is not optimal to reduce their thickness (Table 2(b)). In fact, in this example (i.e. given the maintenance effect and cost parameters), actions that completely or partially renew sections with maximum thickness and reduce their thickness are less efficient than actions that keep the section thicknesses unchanged.

3. When the road section has a 'medium' thickness, there is a trade-off between making the section thicker or making it newer.

Results 1 and 2 confirm expert judgement stating that although the performance of a road is increasing in its thickness, it is not optimal to build the road at it is maximum thickness from the beginning; it is better to add new layers gradually. Moreover, results 1 and 2 indicate 


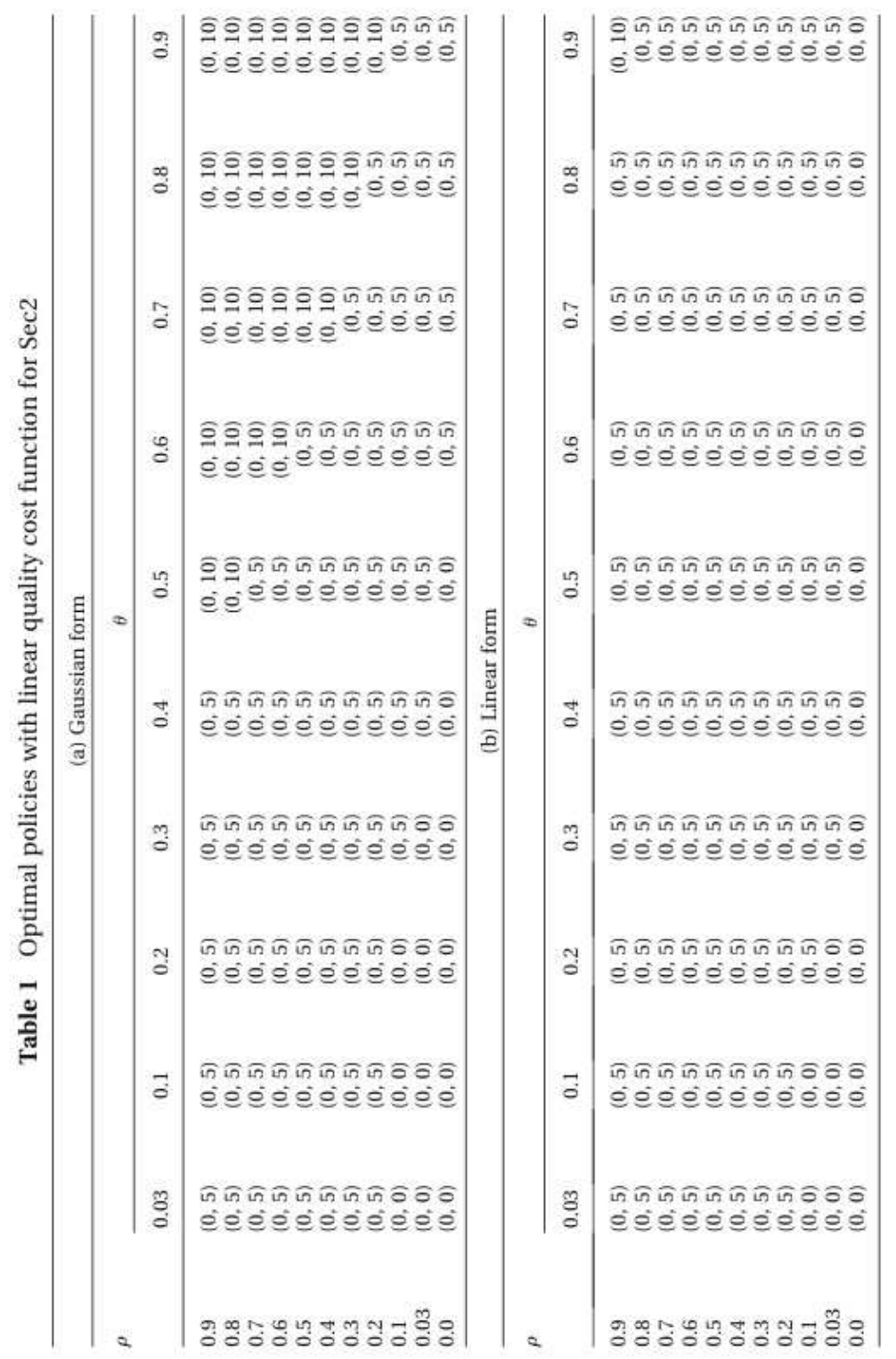




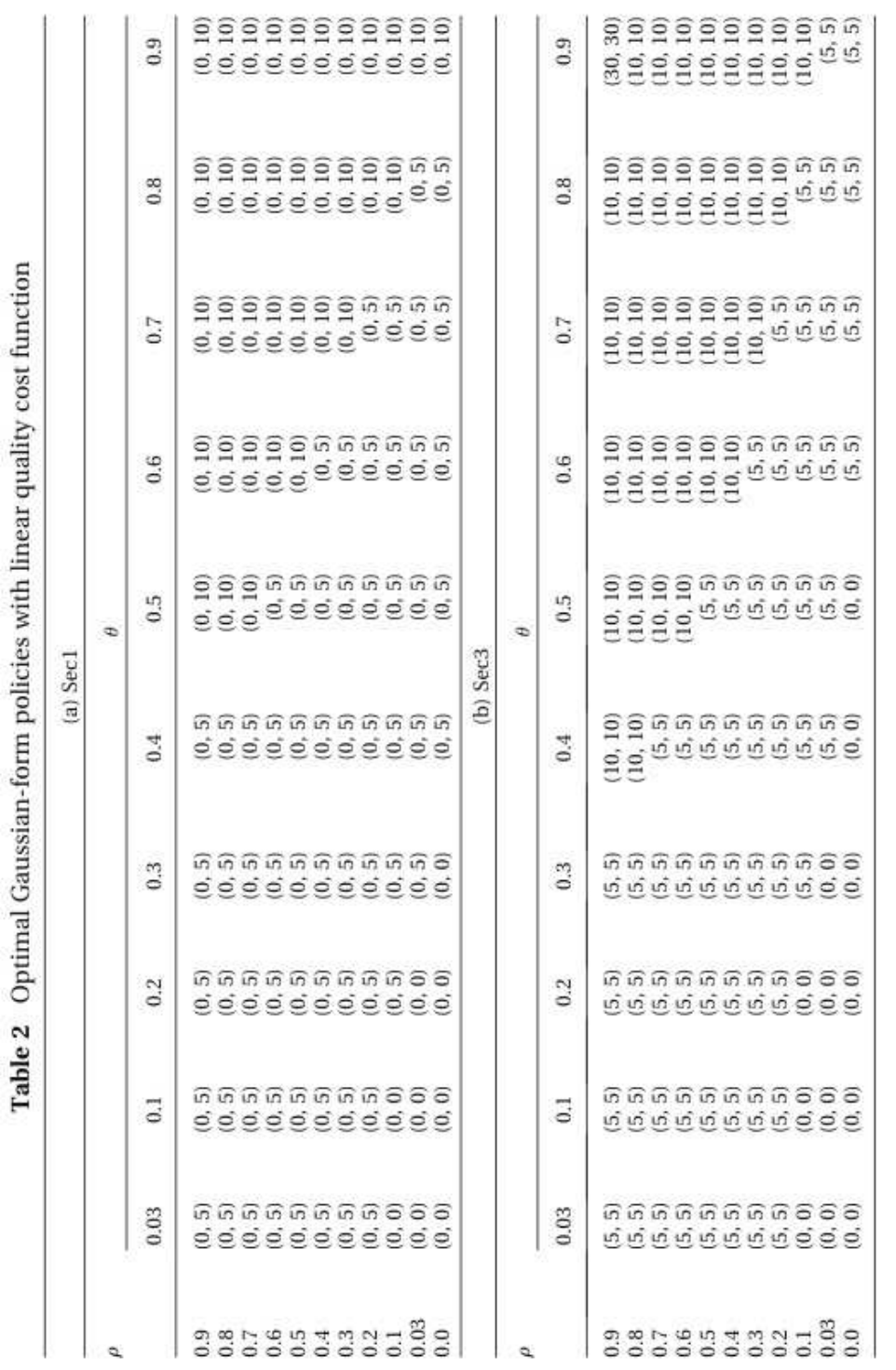


that a control limit in thickness exists under which it is never optimal to reduce the section thickness.

Note that for given unit resurfacing and removing costs, some actions are never selected. This is because action efficiency is determined by the maintenance effect function. If the efficiency order is different from the total cost order, more expensive and less efficient actions will never be selected.

The trade-off between thicker and newer roads (3) is determined by the cost rates. Note that, especially for medium thickness sections, the policies are very sensitive to both maintenance and quality cost variations. The sensitivity of the model to the cost functions is illustrated by Tables 3 and 4, which present policies obtained using the Gaussian-form (a) and linear-form (b) shape functions with different quality cost functions. The policies in Table 3 are obtained using a quality cost function that increases more quickly in $\theta$, whereas the policies in Table 4 are obtained using a quality cost function that increases more quickly in $\rho$.

From the numerical results, the policies are very sensitive to the variations of the quality cost function, especially the Gaussian-form policies. It is found that the Gaussian-form policies are much more sensitive to the quality cost variations in $\theta$ than the linear-form ones. For example, in Table 3, for $\rho>0$, when $\theta$ increases from 0.4 to 0.5, the Gaussian-form policy (a) advises a stronger action than the linear-form one (b), which gives the same action. This confirms the fact that the Gaussian-form shape function takes the effects of the DGR into account better. Note that the actions in Table 3 are ones that make the road thicker, whereas most of the actions in Table 4 keep the section at the same thickness. This reflects the fact that to make roads more rigid, i.e. with lower DGR levels, it is better to make them thicker (Table 3). However, if costs are more sensitive to the LCP variations, it is better to conceal cracks by reducing or keeping the same thickness (Table 4). When the quality cost is not very important compared with the action costs, we note that DN action may become a better choice than maintenance actions that reduce the section thickness, especially for low levels of DGR.

Moreover, Table 4 shows that Gaussian-form policies are more sensitive to the quality cost variations in $\rho$ than linear-form policies. For example, in Table 4 for $\theta>0.5$, when $\rho$ increases from 0.1 to 0.2 , the Gaussian-form policy (a) advises stronger actions whereas the linear-form one (b) gives the same action. This can be explained by the fact that the Gaussian-form shape function represents the effect of the LCP variations on the evolution of the deterioration process better, and therefore reflects the deterioration evolution risk better. Thus the quality cost is a very important function in this model, as it represents a measure of cumulative risk for the decision epoch.

In Tables 5 and 6 , the extended model is compared with the model in reference [13]. However, for the model of reference [13], only resurfacing actions that respect the total maximum thickness constraint are considered. Tables 5 and 6 present policies for low- and high-quality costs compared with maintenance costs, respectively. Note that for quality costs that are less important than maintenance costs, the two approaches converge to the policies presented in Table 5. These two policies have almost the same average values, the extended model allows the section to be renewed to the maximum thickness for maximum deterioration levels. When the deterioration risk is high, i.e. the quality cost is much more important than maintenance 
costs, the two approaches give different policies (Table 6), but the difference in the policy average values is not very significant, i.e. $V(a)=1.05 V(b)$. This shows that the main interest from extending the possible maintenance actions to removing as well as adding different thicknesses is to improve prevention of the cracking risk.

\section{Conclusion}

This paper presents a condition-based maintenance optimization approach for the roadcracking problem based on a Markov decision process. This model extends a previous work [13] by taking into account the constraint of maximum road thickness. The special feature of this approach is that the MDP transition matrix is obtained using a state-dependent deterioration process based on two deterioration parameters, namely the LCP and the DGR, as well as on the road characteristics: the total thickness and the composition in new and old underlying layers. The optimal decision consists in defining both the optimal thickness to remove and the resurfacing thickness to add. This changes the composition of the underlying layers with different mixes of degraded and non-degraded layers and, finally, different cracking patterns. Moreover, we introduce the possibility of actions that can which lead to a state better than new. This approach can be imple- mented directly in a decision tool for designing new roads. The model provides the optimal maintenance costs, given the initial road reliability performance (here defined as a function of the initial road thickness). We have already highlighted the trade-off in initial maximum thickness and maintenance costs.

Moreover, numerical examples show the importance of defining and estimating cost parameters and functions, especially the quality cost function. In fact the quality cost function represents a measure of deterioration risk, and is very important in determining the optimal maintenance to perform. Finally, we provide different numerical analyses of the optimal maintenance policies for different road sections which lead to the identification. All of them lead to the identification of some structural. 


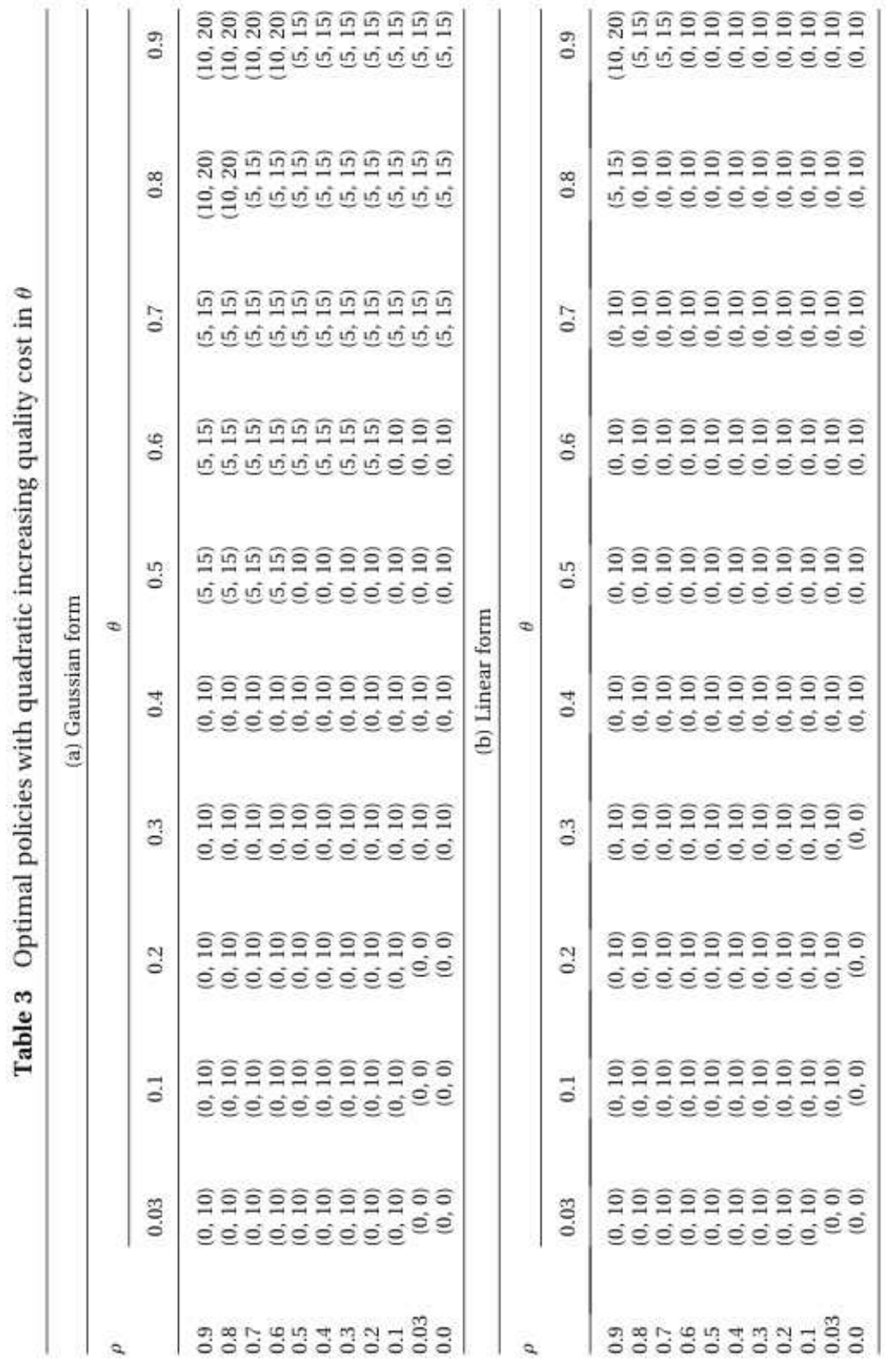




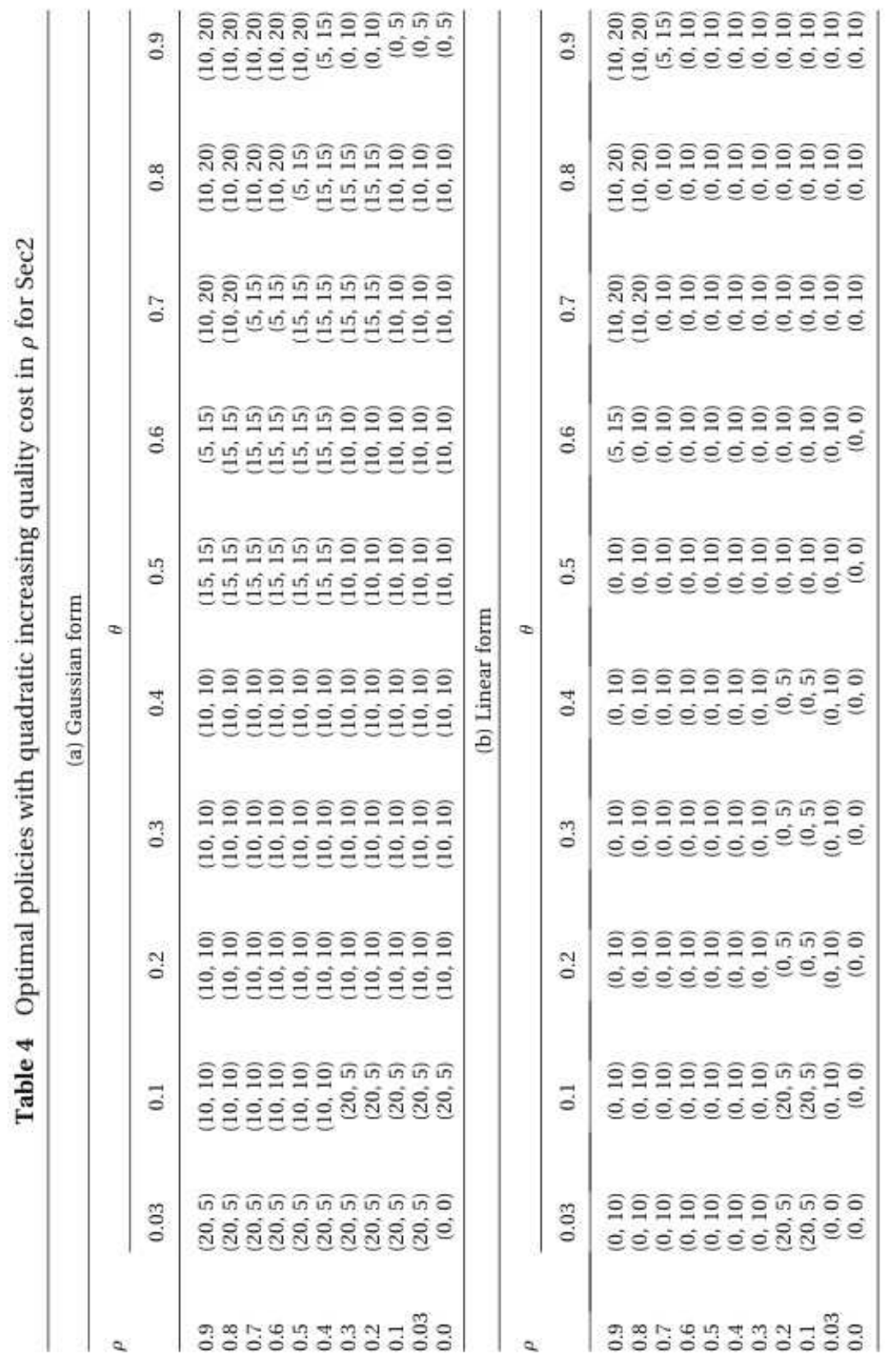




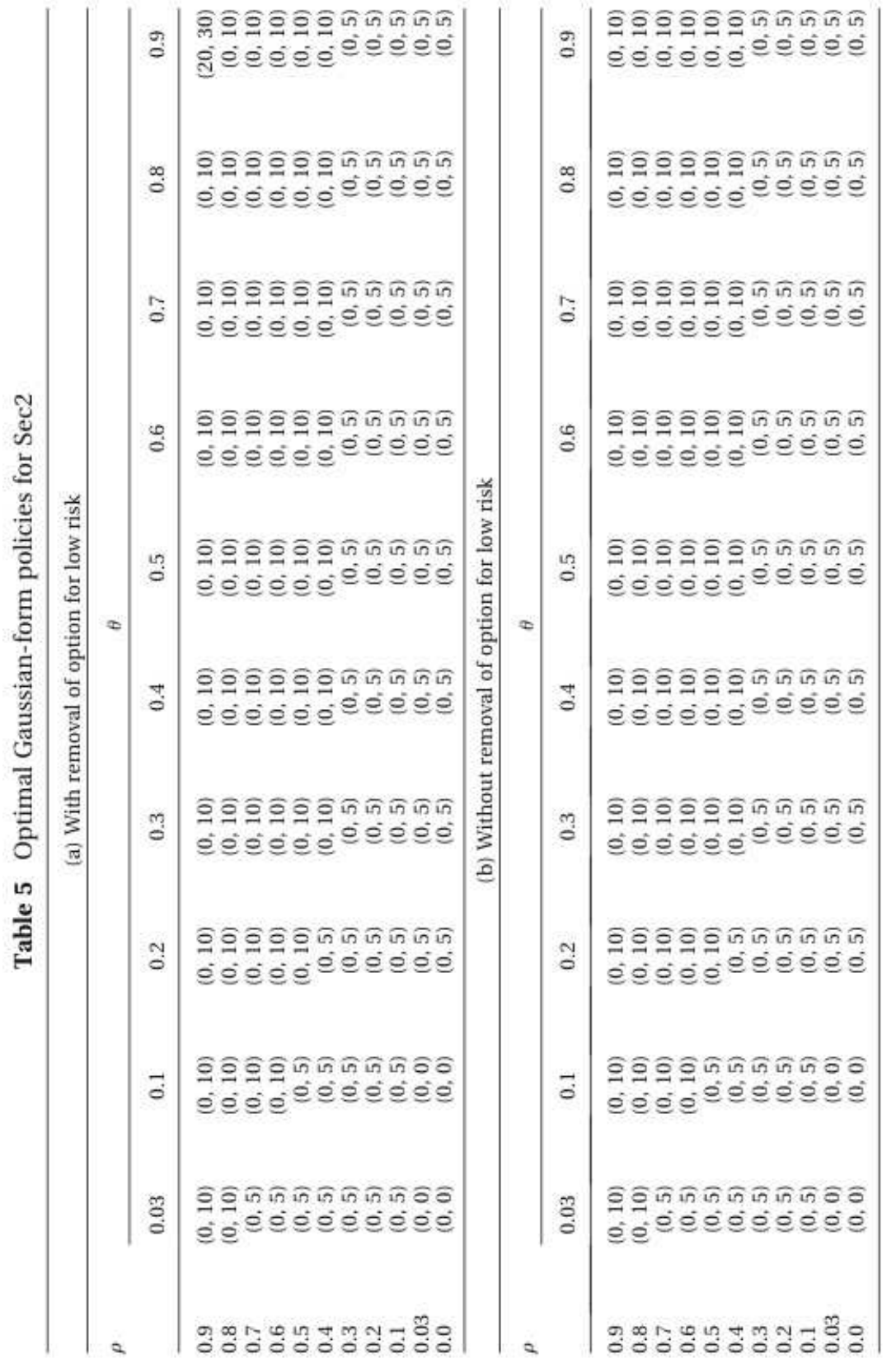




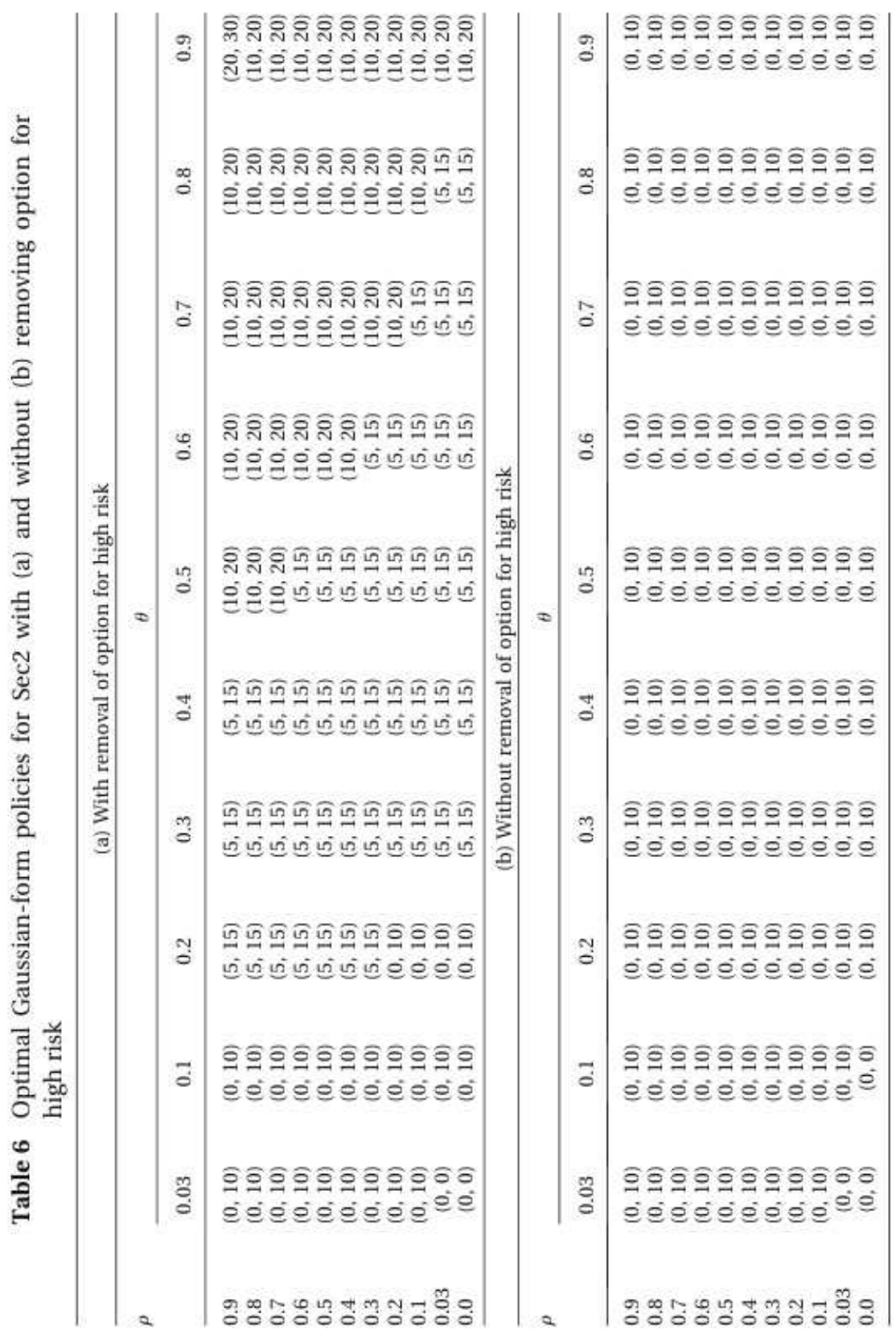




\section{References}

[1] D. M. Frangopol, M. J. Kallen, and J. M. van Noortwijk. Probabilistic models for lifecycle performance of deteriorating structures: review and future directions. Structural Engineering and Materials, 6:197-212, 2004.

[2] N. Ismail, A. Ismail, and R. Atiq. An overview of expert systems in pavement management. European Journal of Scientific Research, 30(1):99-111, 2009.

[3] M. J. Kallen and J. M. van Noortwijk. Optimal periodic inspection of a deterioration process with sequential condition states. International Journal of Pressure Vessels and Piping, 83(4):249 - 255, 2006. The 16th European Safety and Reliability Conference.

[4] L. M. Maillart. Maintenance policies for systems with condition monitoring and obvious failures. IIE Transactions, 38:463-475, 2006.

[5] L. Myers, R. Roque, and B. Ruth. Mechanisms of surface-initiated longitudinal wheel path cracks in high-type bituminous pavements. In Proceedings of Asphalt Paving Technology Conference, volume 67, pages 401-432, 1998.

[6] P. Paris and F. Ergodan. A critical analysis of crack propagation laws. Journal of Basic Engineering, 85(4):528-534, 1963.

[7] M. L. Puterman. Markov decision processes: discrete stochastic dynamic programming. Wiley Series in Probability and Mathematical Statistics: Applied Probability and Statistics. John Wiley and Sons Inc., New York, 1994.

[8] S. G. Ritchie, C. Yeh, J. P. Mahoney, and N. C. Jackson. Surface condition expert system for pavement rehabilitation planning. Journal of Transportation Engineering, 113(2):155-167, 1987.

[9] M. Y. Shahin. Pavement Management For Airport, Roads, and Parking Lots. Chapman and Hall, New York, 1994.

[10] M. Y. Shahin and J. A. Walter. Pavement maintenance management for roads and streets using the paver system. Technical Report technical report M-90/05, US Army Corps of Engineers (USA CERL), Champaign II, 1990.

[11] J. M. van Noortwijk. A survey of the application of gamma processes in maintenance. Reliability Engineering \& System Safety, 94(1):2 - 21, 2009. Maintenance Modeling and Application.

[12] H. Wang. A survey of maintenance policies of deteriorating systems. European Journal of Operational Research, 139(3):469 - 489, 2002.

[13] M. Zouch, T. G. Yeung, and B. Castanier. Two-phase state-dependent deterioration model for maintenance optimization. Submitted to Naval Reasearch Logistics, 2011. 